EDITORIAL

\title{
The value of magnetic resonance guided cardiac catheterisation
}

\author{
V Muthurangu, R S Razavi
}

Magnetic resonance guided cardiac catheterisation, for assessment of physiological parameters, benefits from reduced $x$ ray exposure and more accurate measurement of haemodynamic variables

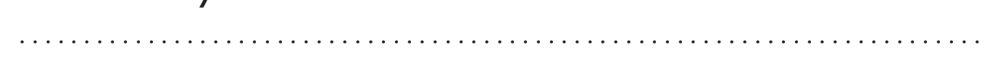

C ardiac magnetic resonance (MR) is increasingly replacing diagnostic cardiac catheterisation as a method of assessing cardiac anatomy, ${ }^{1}$ measuring blood flow, ${ }^{2}$ and quantifying ventricular function, ${ }^{3}$ particularly in patients with congenital heart disease. However, diagnostic cardiac catheterisation remains important when measurement of cardiac or vascular pressures is required. ${ }^{4}$ Patients with pulmonary hypertension, which is characterised by increased pulmonary vascular resistance (PVR), often undergo multiple $x$ ray guided cardiac catheterisations to assess prognosis and response to medical intervention. ${ }^{4}$ Performing such diagnostic cardiac catheterisation procedures under MR guidance has a number of potential benefits, described by Kuehne and colleagues ${ }^{5}$ in this issue of Heart. ${ }^{5}$ MR guided cardiac catheterisation has been a scientific and technical challenge, with a number of groups trying to demonstrate its feasibility in the last few years. The driving force behind this work comprises the potential benefits of this technique, including reduced $x$ ray dose and acquisition of more accurate physiological information.

MR GUIDED CARDIAC CATHETERISATION To make MR guided cardiac catheterisation a reality, there is a need to be able to visualise the catheter within cardiovascular structures in real time. This requires fast imaging, which has become possible because of recent developments in MR hardware and software. Cardiac gated steady state free precession (SSFP) sequences, which benefit from high signal to noise ratio, good blood pool myocardial contrast, and high spatiotemporal resolution, have become the mainstay of cardiac MR. Martin and colleagues ${ }^{6}$ showed that using real time, non-gated SSFP endovascular devices could be easily "passively" visualised. Passive catheter visualisation relies on inherent properties of the catheter to provide either local signal enhancement or loss. In the work of Bakker and colleagues, ${ }^{7}$ dysprosium rings were placed on conventional catheters to create an area of signal loss. We demonstrated that with standard balloon catheters filled with carbon dioxide, passive visualisation and manual tracking of catheters was possible using an
Heart 2005;91:995-996. doi: 10.1136/hrt.2004.055137

interactive SSFP sequence. ${ }^{8}$ The benefit of this approach is that it uses standard catheter laboratory equipment and thus poses no greater safety risk to the patient. There has also been considerable work using active methods of catheter tracking. With these techniques an antenna or coil is incorporated in the catheter allowing automatic tracking and visualisation. ${ }^{9}$ However, there are still some safety concerns with these active tracking techniques because of the possibility of heating.

Over the last few years, there have been a number of studies performed demonstrating the possibility of MR guided cardiac catheterisation in animal models. These studies showed that catheter visualisation and manipulation is possible using both passive and active techniques and can be applied to a number of situations including interventional procedures. ${ }^{10}$

Recently, we published the first study of MR guided cardiac catheterisation in humans. ${ }^{11}$ We were able to demonstrate that MR guided cardiac catheterisation was safe and practical in a clinical setting, with a reduction in ionising radiation exposure, better visualisation of cardiac anatomy, and acquisition of more pertinent physiological information.

\section{REDUCED $X$ RAY EXPOSURE}

Exposure to $x$ rays is increasingly being recognised as a significant health risk for both patients and health care professionals. In adults, the UK National Radiation Protection Board has calculated the mean risk of developing a solid tumour as a result of a single cardiac catheterisation procedure as approximately 1 in $2500 .{ }^{12}$ In children this risk is further increased. This is an important fact as children make up a significant proportion of the population undergoing diagnostic cardiac catheterisation for haemodynamic assessment. The use of wholly MR guided cardiac catheterisation, as in the study by Kuehne and colleagues, ${ }^{5}$ obviously leads to an elimination of $x$ ray exposure. However, in patients with more complex cardiac anatomy or in children it is appropriate to have $x$ ray back up in the same interventional suite, as manipulation may require the use of non-MR compatible catheters or guidewires. ${ }^{11}$ The use of XMR (an interventional MR scanner and $x$ ray fluoroscopy system in a single suite) allows both modalities to be used within a single intervention and still leads to reduced $x$ ray exposure. ${ }^{11}$ Until MR compatible

Abbreviations: $M R$, magnetic resonance; PVR, pulmonary vascular resistance; $P-V$, pressure-volume; SSFP, steady state free precession 
guide wires and catheters with more torque are available we believe that for most patients MR guided catheterisation should be performed using such dual modality systems. Such systems also allow hybrid interventional cardiac catheterisation procedures where MR three dimensional anatomy can be overlaid on to $x$ ray fluoroscopy of non-MR compatible catheters and devices. ${ }^{13}$

\section{IMPROVED PHYSIOLOGICAL INFORMATION}

Cardiac catheterisation is important in the assessment of ventricular and vascular haemodynamics. ${ }^{4}{ }^{14}$ The combination of invasive pressures and blood flow allows calculation of vascular resistance. The calculated PVR, and the response of PVR to nitric oxide and oxygen, are used to assess the prognosis and need for vasodilator treatment in patients with pulmonary hypertension. Unfortunately, the invasive methods of flow quantification that are currently used cannot be considered a "gold standard". The Fick method requires measurement of haemoglobin, aortic and pulmonary artery oxygen saturations and partial pressures, and oxygen consumption, and this dependency on multiple measurements is a considerable source of inaccuracy ${ }^{15}$ Other methods of invasive flow quantification (for example, indicator dilution and thermodilution techniques) also suffer from unreliability, particularly in the presence of valvar regurgitation, which is common in pulmonary hypertensive disease. ${ }^{15}$ There is therefore a need for a method of flow quantification during cardiac catheterisation that allows accurate and reproducible measurement of PVR. Velocity encoded phase contrast MR enables quantification of blood flow in major vessels. Cardiac output and the pulmonary to systemic flow ratio (Qp:Qs) measured using this technique have been shown to be accurate. ${ }^{2}$ In vivo internal validation of phase contrast MR, by comparison of aortic and pulmonary blood flow or comparison of phase contrast flow through the pulmonary artery or aorta with the ventricular output measured from short axis images, has also been performed. We have demonstrated that invasive pressure measurements and MR flow data, obtained during MR guided cardiac catheterisation, can be used to calculate PVR in humans. ${ }^{4}$ This study showed a significant discrepancy between PVR calculated using Fick derived flow and MR derived flow that appeared secondary to inaccuracies in the Fick method. Kuehne and colleagues ${ }^{5}$ have built on this previous work to demonstrate the utility of this MR technique in a homogeneous population of patients with moderate/severe pulmonary hypertension. ${ }^{5}$ In their study they also demonstrated the improved precision of phase contrast MR over thermodilution methods.

MR guided cardiac catheterisation also allows other physiological parameters to be assessed including the construction of pressure-volume (P-V) loops through simultaneous measurement of ventricular volumes and pressures. ${ }^{14}$ It has been shown that cardiac MR is the most accurate and reproducible method of quantifying ventricular volumes ${ }^{3}$ and thus P-V loops constructed using this technique should be able to accurately gauge abnormal ventricular and vascular haemodynamics in pulmonary hypertension.

\section{CONCLUSION}

The feasibility of using MR guided cardiac catheterisation in humans has been demonstrated. However, a number of issues still need to be addressed before MR guided cardiac catheterisation enters widespread clinical use. Further developments by catheter manufacturing companies are needed in order to provide specific products for MR guidance. ${ }^{11}$ Currently, catheters are designed for use in the $x$ ray environment and, consequently, many contain ferromagnetic components (for example, braiding in catheters) or long conducting materials (for example, guidewires) that are potentially hazardous in the MR environment. Non-braided balloon catheters can be used in the MR environment; however, they often lack the desired torque and steerability. In addition, catheter visualisation and tracking must be improved so that the whole length of the catheter can be automatically tracked. This might be achieved using more sophisticated methods of passive tracking ${ }^{16}$ or by the use of safe active catheters. Once these issues have been resolved MR guided cardiac catheterisation will be possible in all patients without $x$ ray back up. The reduced $x$ ray exposure, better anatomical visualisation, and improved accuracy of haemodynamic information gathered should make this the method of choice for diagnostic cardiac catheterisation when haemodynamic data are needed. With further developments in catheters and devices, MR guided cardiac catheterisation may also develop a role in interventional procedures.

\section{Authors' affiliations}

V Muthurangu, R S Razavi, Cardiac MR Research Group, Division of Imaging Sciences, King's College London, London, UK

Sources of funding: Higher Education Funding Council for England Joint Research Equipment Initiative, Philips Medical Systems, Evelina Trust, UK Engineering and Physical Sciences Research Council, and the Charitable Foundation of Guy's and St Thomas Trust.

\section{REFERENCES}

1 Razavi RS, Hill DL, Muthurangu V, et al. Three-dimensional magnetic resonance imaging of congenital cardiac anomalies. Cardiol Young 2003; 13:461-5.

2 Beerbaum P, Korperich H, Barth P, et al. Noninvasive quantification of left-toright shunt in pediatric patients: phase-contrast cine magnetic resonance imaging compared with invasive oximetry. Circulation 2001;103:2476-82.

3 Bellenger NG, Grothues F, Smith GC, et al. Quantification of right and left ventricular function by cardiovascular magnetic resonance. Herz 2000;25:392-9.

4 Muthurangu V, Taylor A, Andriantsimiavona R, et al. Novel method of quantifying pulmonary vascular resistance by use of simultaneous invasive pressure monitoring and phase-contrast magnetic resonance flow. Circulation 2004; 110:826-34.

5 Kuehne T, Yilmaz S, Schulze-Neick I, et al. Magnetic resonance imaging guided catheterisation for assessment of pulmonary vascular resistance: in vivo validation and clinical application in patients with pulmonary hypertension. Heart 2005;91:1064-9.

6 Martin AJ, Weber OM, Saeed M, et al. Steady-state imaging for visualization of endovascular interventions. Magn Reson Med 2003;50:434-8.

7 Bakker CJ, Hoogeveen RM, Hurtak WF, et al. MR-guided endovascular interventions: susceptibility-based catheter and near-real-time imaging technique. Radiology 1997;202:273-6.

8 Miquel ME, Hegde S, Muthurangu V, et al. Visualization and tracking of an inflatable balloon catheter using SSFP in a flow phantom and in the heart and great vessels of patients. Magn Reson Med 2004;51:988-95.

9 Zhang Q, Wendt M, Aschoff AJ, et al. Active MR guidance of interventional devices with target-navigation. Magn Reson Med 2000;44:56-65.

10 Schalla S, Saeed M, Higgins CB, et al. Magnetic resonance-guided cardiac catheterization in a swine model of atrial septal defect. Circulation 2003;108:1865-70.

11 Razavi R, Hill DL, Keevil SF, et al. Cardiac catheterisation guided by MRI in children and adults with congenital heart disease. Lancet 2003;362:1877-82.

12 National Radiation Protection Board. Guidelines on patient dose to promote the optimisation of protection for diagnostic medical exposures. NRPB, 1999.

13 Rhode KS, Hill DL, Edwards PJ, et al. Registration and tracking to integrate Xray and MR images in an XMR facility. IEEE Trans Med Imaging 2003;22:1369-78.

14 Kuehne T, Yilmaz S, Steendijk P, et al. Magnetic resonance imaging analysis of right ventricular pressure-volume loops: in vivo validation and clinical application in patients with pulmonary hypertension. Circulation 2004; 110:2010-6.

15 Hillis LD, Firth BG, Winniford MD. Analysis of factors affecting the variability of Fick versus indicator dilution measurements of cardiac output. Am J Cardiol 1985;56:764-8.

16 Kozerke S, Hegde S, Schaeffter T, et al. Catheter tracking and visualization using 19F nuclear magnetic resonance. Magn Reson Med 2004;52:693-7. 